University of Texas at El Paso

ScholarWorks@UTEP

$2-2004$

\title{
On the Use of Intervals in Scientific Computing: What is the Best Transition from Linear to Quadratic Approximation?
}

\author{
Martine Ceberio \\ The University of Texas at El Paso, mceberio@utep.edu \\ Vladik Kreinovich \\ The University of Texas at El Paso, vladik@utep.edu \\ Lev Ginzburg
}

Follow this and additional works at: https://scholarworks.utep.edu/cs_techrep

Part of the Computer Engineering Commons

Comments:

UTEP-CS-04-07b.

Short version published in Proceedings of the Workshop on State-of-the-Art in Scientific Computing PARA'04, Lyngby, Denmark, June 20-23, 2004, Vol. 1, pp. 43-49; extended version published in Jack Dongarra, Kaj Madsen, and Jerzy Wasniewski (eds.), PARA'04 Workshop on State-of-the-Art in Scientific Computing, Springer Lecture Notes in Computer Science, 2006, Vol. 3732, pp. 75-82.

\section{Recommended Citation}

Ceberio, Martine; Kreinovich, Vladik; and Ginzburg, Lev, "On the Use of Intervals in Scientific Computing: What is the Best Transition from Linear to Quadratic Approximation?" (2004). Departmental Technical Reports (CS). 291.

https://scholarworks.utep.edu/cs_techrep/291

This Article is brought to you for free and open access by the Computer Science at ScholarWorks@UTEP. It has been accepted for inclusion in Departmental Technical Reports (CS) by an authorized administrator of ScholarWorks@UTEP. For more information, please contact Iweber@utep.edu. 


\title{
On the Use of Intervals (and Probabilities) in Scientific Computing: What is the Best Transition from Linear to Quadratic Approximation?
}

\author{
Martine Ceberio $^{1}$, Vladik Kreinovich ${ }^{1}$, and Lev Ginzburg ${ }^{2}$ \\ ${ }^{1}$ Department of Computer Science, University of Texas \\ El Paso, TX 79968, USA, \{mceberio,vladik\}@cs.utep.edu \\ ${ }^{2}$ Department of Ecology and Evolution \\ State University of New York Stony Brook \\ NY 11794, USA, lev@ramas.com, and \\ Applied Biomathematics, 100 N. Country Road \\ Setauket, NY 11733, USA
}

\begin{abstract}
In many problems from science and engineering, the measurements are reasonably accurate, so we can use linearization (= sensitivity analysis) to describe the effect of measurement errors on the result of data processing.

In many practical cases, the measurement accuracy is not so good, so, to get a good estimate of the resulting error, we need to take quadratic terms into consideration - i.e., in effect, approximate the original algorithm by a quadratic function. The problem of estimating the range of a quadratic function is NP-hard, so, in the general case, we can only hope for a good heuristic.

Traditional heuristic is similar to straightforward interval computations: we replace each operation with numbers with the corresponding operation of interval arithmetic (or of the arithmetic that takes partial probabilistic information into consideration). Alternatively, we can first diagonalize the quadratic matrix - and then apply the same approach to the result of diagonalization.

Which heuristic is better? We show that sometimes, the traditional heuristic is better; sometimes, the new approach is better; asymptotically, which heuristic is better depends on how fast, when sorted in decreasing order, the eigenvalues decrease.
\end{abstract}




\section{Formulation of the Problem}

Need for data processing and indirect measurements in scientific computing. In many areas of science and engineering, we are interested in the value of a physical quantity $y$ that is difficult (or even impossible) to measure directly. Examples may include the amount of a pollutant in a given lake, the distance to a faraway star, etc.

To measure such quantities, we find auxiliary easier-to-measure quantities $x_{1}, \ldots, x_{n}$ that are related to $y$ by a known algorithm $y=f\left(x_{1}, \ldots, x_{n}\right)$. In some cases, the relation between $x_{i}$ and $y$ is known exactly. In such cases, to estimate $y$, we measure $x_{i}$, and apply the algorithm $f$ to the results $\widetilde{x}_{1}, \ldots, \widetilde{x}_{n}$ of measuring $x_{i}$. As a result, we get an estimate $\widetilde{y}=f\left(\widetilde{x}_{1}, \ldots, \widetilde{x}_{n}\right)$ for $y$.

In many other practical situations, we only know an approximate relation $y \approx \widetilde{f}\left(x_{1}, \ldots, x_{n}\right)$, with an upper bound $\varepsilon_{f}$ on the accuracy of this approximation:

$$
\left|\widetilde{f}\left(x_{1}, \ldots, x_{n}\right)-f\left(x_{1}, \ldots, x_{n}\right)\right| \leq \varepsilon_{f} .
$$

In such cases, to estimate $y$, we measure $x_{i}$, and apply the algorithm $\widetilde{f}$ to the results $\widetilde{x}_{1}, \ldots, \widetilde{x}_{n}$ of measuring $x_{i}$. As a result, we get an estimate $\widetilde{y}=$ $\widetilde{f}\left(\widetilde{x}_{1}, \ldots, \widetilde{x}_{n}\right)$ for $y$.

This indirect measurement (data processing) is one of the main reasons why computers were invented in the first place, and one of the main uses of computers is scientific computing.

Need for error estimation for indirect measurements in scientific computing. Measurements are never $100 \%$ accurate. The results $\widetilde{x}_{i}$ of direct measurements are, in general, different from the actual values $x_{i}$. Therefore, the estimate $\widetilde{y}=f\left(\widetilde{x}_{1}, \ldots, \widetilde{x}_{n}\right)$ is, in general, different from the actual (unknown) value $y=f\left(x_{1}, \ldots, x_{n}\right)$. What do we know about the error $\Delta y \stackrel{\text { def }}{=} \widetilde{y}-y$ of the indirect measurement?

Estimating errors of indirect measurements: formulation of the problem. In many cases, we know the upper bounds $\Delta_{i}$ on the measurement errors $\Delta x_{i} \stackrel{\text { def }}{=} \widetilde{x}_{i}-x_{i}$ of direct measurements. Once we know such an upper bound, we can guarantee that the actual value $x_{i}$ lies in the interval $\mathbf{x}_{i} \stackrel{\text { def }}{=}$ $\left[\widetilde{x}_{i}-\Delta_{i}, \widetilde{x}_{i}+\Delta_{i}\right]$. In this case, if we know the relation $y=f\left(x_{1}, \ldots, x_{n}\right)$ exactly, then the only information that we have about $y$ is that $y$ belongs to the range $[\underline{r}, \bar{r}] \stackrel{\text { def }}{=} f\left(\mathbf{x}_{1}, \ldots, \mathbf{x}_{n}\right)$.

In situations when, instead of knowing the exact relation $y=f\left(x_{1}, \ldots, x_{n}\right)$, we only know:

- the approximate relation $y \approx \widetilde{f}\left(x_{1}, \ldots, x_{n}\right)$ between $x_{i}$ and $y$ and

- we know the upper bound $\varepsilon_{f}$ on the accuracy of approximating $f$ by $\tilde{f}$, 
then we can guarantee that $y$ belongs to the interval $\left[\underline{r}-\varepsilon_{f}, \bar{r}+\varepsilon_{f}\right]$, where $[\underline{r}, \bar{r}] \stackrel{\text { def }}{=} \widetilde{f}\left(\mathbf{x}_{1}, \ldots, \mathbf{x}_{n}\right)$ is the range of a known algorithmic function $\widetilde{f}\left(x_{1}, \ldots, x_{n}\right)$ on the "box" $\mathbf{x}_{1} \times \ldots \times \mathbf{x}_{n}$.

In both cases, to find the range of possible values of $y$, we must find the range $[\underline{r}, \bar{r}]$ of a known algorithmic function $f$ (or $\widetilde{f}$ ) on the known box.

Comment. In some engineering situations, instead of knowing the guaranteed upper bounds $\Delta_{i}$ on the measurement errors, we only have estimates $\Delta_{i}$ of the upper bounds. In such situations, it is still desirable to compute the corresponding range for $y$ - but we can no longer absolutely guarantee that the actual value $y$ belong to the resulting range; we can only guarantee it under the condition that the estimates are correct.

Interval computations: a way to estimate errors of indirect measurements. Interval computations enable us to either compute the range a given algorithmic function $f$ (or $\widetilde{f}$ ) on the given box exactly, or at least to provide an enclosure for this range. For the case when $n=2$ and the function $f\left(x_{1}, x_{2}\right)$ is one of the standard arithmetic operations $(+,-$, multiplication, etc. $)$, there are known explicit formulas for the range of $f$. For example,

$$
\left[\underline{x}_{1}, \bar{x}_{1}\right]+\left[\underline{x}_{2}, \bar{x}_{2}\right]=\left[\underline{x}_{1}+\underline{x}_{2}, \bar{x}_{1}+\bar{x}_{2}\right] .
$$

These formulas form interval arithmetic; see, e.g., [5, 6, 12].

One way to compute the range for more complex functions $f$ is to use straightforward ("naive") interval computations, i.e., replace each operation forming the algorithm $f$ with the corresponding operation from interval arithmetic. This technique leads to an interval that is guaranteed to be an enclosure, i.e., to contain the desired range, but it is known that this interval contains $e x$ cess width, i.e., is wider than the desired range $[5,6,12]$. How can we reduce this excess width?

When measurement errors are small, linearization works well. When the measurement errors $\Delta x_{i}$ are relatively small, we can expand $f$ into Taylor series in terms of $\Delta x_{i}$ and ignore quadratic and higher terms - i.e., keep only linear terms. In a linear expression $f=a_{0}+a_{1} \cdot \Delta x_{1}+\ldots+a_{n} \cdot \Delta x_{n}$, each variable $\Delta x_{i} \in\left[-\Delta_{i}, \Delta_{i}\right]$ occurs only once. It is known that for such singleuse expressions (SUE), straightforward interval computations leads to the exact range; see, e.g., $[3,5]$.

Quadratic approximation is more difficult to analyze. In many real-life situations, measurement errors $\Delta x_{i}$ are not so small, so we must also take into 
consideration terms that are quadratic in $\Delta x_{i}$. So, we must be able to estimate the range of a quadratic function

$$
f=a_{0}+\sum_{i=1}^{n} a_{i} \cdot \Delta x_{i}+\sum_{i=1}^{n} \sum_{j=1}^{n} a_{i j} \cdot \Delta x_{i} \cdot \Delta x_{j},
$$

or, equivalently,

$$
f=a_{0}+\sum_{i=1}^{n} a_{i} \cdot \Delta x_{i}+\sum_{i=1}^{n} a_{i i} \cdot\left(\Delta x_{i}\right)^{2}+\sum_{i=1}^{n} \sum_{j \neq i} a_{i j} \cdot \Delta x_{i} \cdot \Delta x_{j} .
$$

There exist methods for computing the exact range of such a function (see, e.g., [6]), but all such methods require $2^{n}$ steps - the number of steps which, even for a realistically large number of inputs $n \approx 10^{2}-10^{3}$, can be impossibly large. Since the problem of estimating range of a given quadratic function is, in general, NP-hard (see, e.g., $[10,15]$ ), we cannot hope to get an algorithm that is always faster. So, for large $n$, we can only compute enclosures.

Two natural approaches to compute enclosure: which is better? One approach to computing the enclosure of a quadratic approximation function (1) is to use naive (straightforward) interval computations. As we have mentioned, in this approach, we often get excess width.

There is a particular case when we do not have any excess width - when the matrix $A=\left(a_{i j}\right)_{i, j}$ is diagonal. In this case, $f$ can be represented as a sum of the terms $a_{i} \cdot \Delta x_{i}+a_{i i} \cdot \Delta x_{i}^{2}$ corresponding to different variables, and each of these terms can be reformulated as a SUE expression $a_{i i} \cdot\left(\Delta x_{i}+a_{i} /\left(2 a_{i i}\right)\right)^{2}+$ const - thus making the whole expression SUE.

Every quadratic function can be represented in a similar diagonal form as a linear combination of squares of eigenvectors. It therefore seems reasonable to first represent a quadratic function in this form, and only then apply straightforward interval computations.

A natural question is: which approach is better? If none of them is always better, then when is the first approach better and when is the second one better?

Beyond interval computations: towards joint use of probabilities and intervals in scientific computing. In many cases, in addition to the upper bounds on $\Delta x_{i}$, we have partial information on the probabilities of different values of $\Delta x \stackrel{\text { def }}{=}\left(\Delta x_{1}, \ldots, \Delta x_{n}\right)$.

In particular, in some applications, we know that the input variables $x_{i}$ are not truly independent and are in fact correlated. This knowledge about correlation is also usually represented in the probabilistic terms, as partial information about the probability distribution of $\Delta x$.

In all such cases, in addition to the interval range, we would like to compute the information about the probabilities of different values of $y$. There exist ways 
of extending interval arithmetic to such cases; see, e.g., [1]. We can therefore use both approaches in these cases as well.

What we are planning to do. In this paper, we show that which method is better depends on the eigenvalues of the matrix $B=\left(a_{i j} \cdot \Delta_{i} \cdot \Delta_{j}\right)_{i, j}$ : on average, the eigenvector method is better if and only if the eigenvalues (when sorted in decreasing order) decrease fast enough.

\section{Formalizing the Problem in Precise Terms}

Simplifying the problem. Let us start by simplifying the above problem.

In the original formulation of the problem, we have parameters $a_{0}, a_{i}$, and $a_{i j}$ that describe the function $f$ and the parameters $\Delta_{i}$ that describe the accuracy of measuring each of $n$ variables. We can reduce the number of parameters if we re-scale each of $n$ variables in which a way that $\Delta_{i}$ becomes 1 . Indeed, instead of the variables $\Delta x_{i}$, let us introduce the new variables $y_{i} \stackrel{\text { def }}{=} \Delta x_{i} / \Delta_{i}$. For each of $y_{i}$, the interval of possible values is $[-1,1]$. Substituting $\Delta x_{i}=\Delta_{i} \cdot y_{i}$ into the expression (1), we get the expression for $f$ in terms of $y_{i}$ :

$$
f=b_{0}+\sum_{i=1}^{n} b_{i} \cdot y_{i}+\sum_{i=1}^{n} b_{i i} \cdot y_{i}^{2}+\sum_{i=1}^{n} \sum_{j \neq i} b_{i j} \cdot y_{i} \cdot y_{j},
$$

where $b_{0} \stackrel{\text { def }}{=} a_{0}, b_{i} \stackrel{\text { def }}{=} a_{i} \cdot \Delta_{i}$, and $b_{i j} \stackrel{\text { def }}{=} a_{i j} \cdot \Delta_{i} \cdot \Delta_{j}$.

In the following text, we will therefore assume that $\Delta_{i}=1$ and that the quadratic form has the form (2).

Explicit expressions for the results of the two compared methods. Let us explicitly describe the results of applying the two methods to the quadratic form (2).

If we directly apply straightforward interval computations to the original expression (2), then, since $y_{i} \in[-1,1]$, we get the enclosure $f^{(0)}+\mathbf{f}^{(1)}+\mathbf{f}_{\text {orig }}^{(2)}$, where $f^{(0)}=b_{0}, \mathbf{f}^{(1)}=\left[-\sum_{i=1}^{n}\left|b_{i}\right|, \sum_{i=1}^{n}\left|b_{i}\right|\right]$, and

$$
\mathbf{f}_{\text {orig }}^{(2)}=\sum_{i=1}^{n}\left(b_{i i} \cdot[0,1]\right)+\sum_{i=1}^{n} \sum_{j \neq i}\left|b_{i j}\right| \cdot[-1,1]
$$

Alternatively, we can represent the matrix $B=\left(b_{i j}\right)_{i, j}$ in terms of its eigenvalues $\lambda_{k}$ and the corresponding unit eigenvectors $e_{k}=\left(e_{k 1}, \ldots, e_{k n}\right)$, as

$$
b_{i j}=\sum_{k=1}^{n} \lambda_{k} \cdot e_{k i} \cdot e_{k j}
$$


In this case, the original expression (2) takes the form

$$
b_{0}+\sum_{i=1}^{n} b_{i} \cdot y_{i}+\sum_{k=1}^{n} \lambda_{k} \cdot\left(\sum_{i=1}^{n} e_{k i} \cdot y_{i}\right)^{2} .
$$

Since $y_{i} \in[-1,1]$, we conclude that $\sum_{i=1}^{n} e_{k i} \cdot y_{i} \in\left[-B_{k}, B_{k}\right]$, where $B_{k} \stackrel{\text { def }}{=} \sum_{i=1}^{n}\left|e_{k i}\right|$. Therefore, $\left(\sum_{i=1}^{n} e_{k i} \cdot y_{i}\right)^{2} \in\left[0, B_{k}^{2}\right]$, and so, when applied to the expression (5), straightforward interval computations lead to the expression $f^{(0)}+\mathbf{f}^{(1)}+\mathbf{f}_{\text {new }}^{(2)}$, in which linear terms $f^{(0)}$ and $\mathbf{f}^{(1)}$ are the same, while

$$
\mathbf{f}_{\text {new }}^{(2)}=\sum_{k=1}^{n} \lambda_{k} \cdot\left[0,\left(\sum_{i=1}^{n}\left|e_{k i}\right|\right)^{2}\right]
$$

So, to decide which method is better, it is sufficient to consider only quadratic terms.

Example when the eigenvalue-related expression is better. If the matrix $B$ has only one non-zero eigenvector $\lambda_{1} \neq 0$, then the formula (5) takes a simplified form: $\lambda_{1} \cdot\left(\sum_{i=1}^{n} e_{1 i} \cdot y_{i}\right)^{2}$. This is a SUE expression, so straightforward interval computations lead to the exact range.

For such matrices, the original expression (1) is not necessarily SUE, and may lead to excess width. For example, for a $2 \times 2$ matrix with $b_{i j}=1$ for all $i$ and $j$, the only non-zero eigenvalue is $\lambda_{1}=2$ (with eigenvector $(1,1)$ ). So, the new expression leads to the exact range $[0,4]$. On the other hand, if we apply straightforward interval computations to the original expression (2), then the resulting expression (3) leads to $[-2,4]$, i.e., to excess width.

Example when the original expression is better. For the identity matrix $B$, the original quadratic expression (2) leads to a SUE expression $\sum b_{i i} \cdot\left(\Delta x_{i}\right)^{2}$ for which straightforward interval computations lead to the exact range. For example, for $n=1$, we get the range $[0,2]$.

On the other hand, if we select eigenvectors that are different from $(1,0)$ and $(0,1)$, we may get excess width. For example, if we choose $e_{1}=(\sqrt{2} / 2, \sqrt{2} / 2)$ and $e_{2}=(\sqrt{2} / 2,-\sqrt{2} / 2)$, then, for straightforward interval computations, the range of $\frac{\sqrt{2}}{2} \Delta x_{1}+\frac{\sqrt{2}}{2} \Delta x_{2}$ is $[-\sqrt{2}, \sqrt{2}]$, hence the range of its square is $[0,2]$, and the range of the resulting quadratic expression is estimated as $[0,4]$. 
How do we compare different approaches: randomization needed. The main difference between the two cases is in the eigenvalues of the matrix $B$ : In the first example, we had only one non-zero eigenvalue, and the eigenvaluerelated expression leads to better estimates. In the second example, we have equal eigenvalues, and the original expression is better. It is therefore natural to assume that which method is better depends on the eigenvalues $\lambda_{k}$ of the matrix $B$.

We should not expect a result of the type "if we have certain $\lambda_{k}$, then the first method is always better" - which method is better depends also on the eigenvectors. For example, in the second case, if we select $(1,0)$ and $(0,1)$ as eigenvectors, then the eigenvalue-related expression also leads to the same optimal range estimate. In other words, for a given set of eigenvalues $\lambda_{k}$, we should not expect a result saying that one of the methods is better for all possible eigenvectors: for some eigenvectors the first methods will be better, for some others the second method will be better. In such a situation, it is reasonable to analyze which method is better on average, if we consider random eigenvectors.

Natural probability measure on the set of all eigenvectors. What is the natural probability measure on the set of all possible eigenvectors $e_{1}, \ldots, e_{n}$ ? In general, we have $n$ mutually orthogonal unit vectors, i.e., an orthonormal base in the $n$-dimensional space. It is reasonable to assume that the probability distribution on the set of all such bases is rotation-invariant. This assumption uniquely determines the probability distribution; see, e.g., [7, 14].

Indeed, the first unit vector $e_{1}$ can be uniquely represented by its endpoint on a unit sphere. The only possible rotation-invariant distribution on a unit sphere is a uniform distribution. Once $e_{1}$ is fixed, $e_{2}$ can be any vector from a sphere in an $(n-1)$-dimensional space of all vectors orthogonal to $e_{1}$; the only rotation-invariant distribution on this sphere is also uniform, etc. So, in the resulting distribution, $e_{1}$ is selected from the uniform distribution on the unit sphere, $e_{2}$ from the uniform distribution on the unit sphere in the subspace of all vectors $\perp e_{1}$, etc.

\section{Main Result}

Theorem 1 When $n \rightarrow \infty$, then asymptotically, the expected values are:

$$
\begin{gathered}
E\left[\mathbf{f}_{\text {orig }}^{(2)}\right] \sim\left[-\sqrt{\frac{2}{\pi}} \cdot n \cdot \sqrt{\sum_{k=1}^{n} \lambda_{k}^{2}}, \sqrt{\frac{2}{\pi}} \cdot n \cdot \sqrt{\sum_{k=1}^{n} \lambda_{k}^{2}}\right] ; \\
E\left[\mathbf{f}_{\text {new }}^{(2)}\right] \sim\left[\frac{2}{\pi} \cdot n \cdot \sum_{k: \lambda_{k}<0} \lambda_{k}, \frac{2}{\pi} \cdot n \cdot \sum_{k: \lambda_{k}>0} \lambda_{k}\right] .
\end{gathered}
$$


Conclusions. If $\sum\left|\lambda_{k}\right|<\sqrt{\pi / 2} \cdot \sqrt{\sum \lambda_{k}^{2}}$, then asymptotically, $E\left[\mathbf{f}_{\text {new }}^{(2)}\right] \subset$ $E\left[\mathbf{f}_{\text {orig }}^{(2)}\right]$, so the eigenvector-based method is definitely better.

If $\sum\left|\lambda_{k}\right|<\sqrt{2 \pi} \cdot \sqrt{\sum \lambda_{k}^{2}}$, then the interval $E\left[\mathbf{f}_{\text {new }}^{(2)}\right]$ is narrower than $E\left[\mathbf{f}_{\text {orig }}^{(2)}\right]$, so in this sense, the new method is also better.

Example. The spectrum $\lambda_{k}$ often decreases according to the power law $\lambda_{k} \sim$ $k^{-\alpha}$. In this case, $\sum\left|\lambda_{k}\right| \approx \int_{1}^{\infty} x^{-\alpha} \mathrm{d} x=1 /(\alpha-1)$ and $\sum \lambda_{k}^{2} \approx \int_{1}^{\infty} x^{-2 \alpha} \mathrm{d} x=$ $1 /(2 \alpha-1)$, so the above inequality turns into $(\alpha-1)^{2} \geq(2 / \pi) \cdot(2 \alpha-1)$, which is equivalent to

$$
\alpha \geq 1+\frac{2}{\pi}+\sqrt{\left(1+\frac{2}{\pi}\right) \cdot \frac{2}{\pi}} \approx 2.7 .
$$

Hence, if the eigenvalues decrease fast $(\alpha \geq 2.7)$, the new method is definitely better. For $\alpha \geq 1.6$, the new method leads to narrower intervals; otherwise, the traditional method leads, on average, to better estimates.

Proof of Theorem 1. Before we start the proof, let us derive some auxiliary formulas. Since each vector $e_{k}$ is a unit vector, we have $\sum_{i} e_{k i}^{2}=1$. Due to rotation invariance, the expected value $E\left[e_{k i}^{2}\right]$ should not depend on $i$, hence $E\left[e_{k i}^{2}\right]=1 / n$. Similarly, from $\sum_{i} e_{k i} \cdot e_{l i}=0$ and rotation invariance, we conclude that $E\left[e_{k i} \cdot e_{l i}\right]=0$.

For given $k, l$, and $i \neq j$, the value $E\left[e_{k i} \cdot e_{l j}\right]$ should not change under the transformation $x_{i} \rightarrow x_{i}$ and $x_{j} \rightarrow-x_{j}$, so $E\left[e_{k i} \cdot e_{l j}\right]=0$.

To compute $E\left[\mathbf{f}_{\text {orig }}^{(2)}\right]$, we must find $E\left[b_{i i}\right]$ and $E\left[\left|b_{i j}\right|\right]$. By definition (4), for each $i, E\left[b_{i i}\right]=\sum_{k} \lambda_{k} \cdot E\left[e_{k i}^{2}\right]=(1 / n) \cdot \sum \lambda_{k}$, so the sum of $n$ such terms is proportional to $\sum \lambda_{k}$.

For $i \neq j$, due to the central limit theorem, the distribution for $b_{i j}$ (formula (4)) is asymptotically Gaussian, so asymptotically, $E\left[\left|b_{i j}\right|\right] \sim \sqrt{2 / \pi} \cdot \sqrt{E\left[b_{i j}^{2}\right]}$. Here, $E\left[b_{i j}^{2}\right]=\sum_{k} \sum_{l} \lambda_{k} \cdot \lambda_{l} \cdot E\left[e_{k i} \cdot e_{k j} \cdot e_{l i} \cdot e_{l j}\right]$. Due to symmetry, each $k \neq l$ term is 0 , so $E\left[b_{i j}^{2}\right]=\sum_{k} \lambda_{k}^{2} \cdot E\left[e_{k i}^{2} \cdot e_{k j}^{2}\right]$. Asymptotically, $e_{k i}$ and $e_{k j}$ are independent, so $E\left[e_{k i}^{2} \cdot e_{k j}^{2}\right] \sim E\left[e_{k i}^{2}\right] \cdot E\left[e_{k j}^{2}\right]=(1 / n)^{2}$. Therefore, $E\left[b_{i j}^{2}\right] \sim(1 / n)^{2} \cdot \sum \lambda_{k}^{2}$, hence $E\left[\left|b_{i j}\right|\right] \sim \sqrt{2 / n} \cdot(1 / n) \cdot \sqrt{\sum \lambda_{k}^{2}}$. The sum of $n(n-1)$ such terms is $\sim \sqrt{2 / \pi} \cdot n \cdot \sqrt{\lambda_{k}^{2}}$. The sum of the terms $E\left[b_{i i}\right]$ is asymptotically smaller, so when $n \rightarrow \infty$, we get the expression (7).

For the new expression, we must compute, for every $k$, the expected value of $E\left[\left(\sum_{i}\left|e_{k i}\right|\right)^{2}\right]=\sum_{i, j} E\left[\left|e_{k i}\right| \cdot\left|e_{k j}\right|\right]$. Asymptotically, $e_{k i}$ and $e_{k j}$ are inde- 
pendent, and $E\left[\left|e_{k i}\right|\right] \sim \sqrt{2 / \pi} \cdot \sqrt{E\left[e_{k i}^{2}\right]}=\sqrt{2 / \pi} \cdot(1 / \sqrt{n})$. Thus, the sum of all the terms $i \neq j$ is $\sim n^{2} \cdot(2 / \pi) \cdot(1 / n)=(2 / \pi) \cdot n$. The terms with $i=j$ are asymptotically smaller, so we get the desired expression (8). The theorem is proven.

\section{Auxiliary Result: Situations When We Also Have Partial Information About Probabilities}

Motivations. As we have mentioned, in many cases, in addition to the upper bounds on the measurement errors $\Delta x_{i}$, we have partial information on the probabilities of different values of $\Delta x \stackrel{\text { def }}{=}\left(\Delta x_{1}, \ldots, \Delta x_{n}\right)$.

For example, in addition to the interval range of each variable $\Delta x_{i}$, we often know the mean $\Delta E_{i}$ of $\Delta x_{i}$. We get it, e.g., from the results of the testing the measuring instrument, when the mean is estimated as the average of measurement errors. The more tests we undertake, the more information we get about the probability distribution, and the more characteristics of the probability distribution we can determine. In the ideal situation, we can perform as many tests as necessary to determine the probability distribution of $\Delta x_{i}$. In many real-life situations, however, we can only afford to determine one (or two) characteristics. In such situations, a natural choice is to determine the mean (and, if possible, the standard deviation; see, e.g., [13]).

In measurement terms, the difference between the mean and the actual value of the measured quantity is called the systematic error of the measurement procedure. In measurements, it is a common practice to calibrate the measuring instrument so that the systematic error (bias) is eliminated. Calibration means that, instead of the original measured value $\widetilde{x}_{i}$ of the desired property, we return the value $E_{i} \stackrel{\text { def }}{=} \widetilde{x}_{i}-\Delta E_{i}$ for which the mean value of the re-calibrated measurement error $\Delta x_{i}^{\prime} \stackrel{\text { def }}{=} E_{i}-x_{i}$ is exactly 0 .

The original measurement error $\Delta x_{i}$ can attain any value from the interval $\left[-\Delta_{i}, \Delta_{i}\right]$. As a result, the re-calibrated measurement error $\Delta_{i}^{\prime}$ can take all possible values from the interval $\left[-\Delta_{i}^{-}, \Delta_{i}^{+}\right]$, where $\Delta_{i}^{-} \stackrel{\text { def }}{=} \Delta_{i}+\Delta E_{i}$ and $\Delta_{i}^{+} \stackrel{\text { def }}{=}$ $\Delta_{i}-\Delta E_{i}$.

For example, if we know that $\Delta x_{i} \in[-0.1,0.1]$, and its mean is $\Delta E_{i}=0.05$, then for $\Delta x_{i}^{\prime}=\Delta x_{i}-\Delta E_{i}$, the mean is 0 , and the interval of possible values is $[-0.15,0.05]$.

The mean is the only information that we have about each measurement error. What do we know about the dependence between the corresponding random variables? In many applications, we know that the same source of noise contributes to the errors of different measurements, so these errors $\Delta x_{i}$ are not independent. Since we do not have enough statistics to get any information about each distribution except for its mean, we also cannot determine the correlation between $\Delta x_{i}$. So, if we are interested in guaranteed estimates, we must 
consider all possible $n$-dimensional distributions, with all possible correlations.

Formulation of the problem. In the case of interval uncertainty, we know the intervals of possible values of the measurement errors $\Delta x_{i}$, and we want to find the interval of possible values of the desired quantity $y=f\left(x_{1}, \ldots, x_{n}\right)$.

In the situations when, in addition to the interval of possible values of measurement error, we also know the mean of the measurement errors, in addition to knowing the interval of possible values for the result $y$ of data processing, it is desirable to also know the interval of possible values for the the mean $E \stackrel{\text { def }}{=} E[y]$ of the quantity $y$.

In this paper, we consider the case when the dependence $f\left(x_{1}, \ldots, x_{n}\right)$ between the desired quantity $y$ and the directly measurable quantities $x_{1}, \ldots, x_{n}$ can be safely described by a quadratic function (1). So, the problem is: we know the intervals $\left[-\Delta_{i}, \Delta_{i}\right]$ of possible values of $\Delta x_{i}$, we know the means $\Delta E_{i}=E\left[\Delta x_{i}\right]$, we want to estimate the mean $E[y]$ of the expression (1).

Alternatively, if we re-calibrate all the measuring instruments and take the re-calibrated measured values $E_{i}=\widetilde{x}_{i}-\Delta E_{i}$, then we can expand $f$ around these new values $E_{i}$ :

$$
f=a_{0}^{\prime}+\sum_{i=1}^{n} a_{i}^{\prime} \cdot \Delta x_{i}^{\prime}+\sum_{i=1}^{n} a_{i i}^{\prime} \cdot\left(\Delta x_{i}^{\prime}\right)^{2}+\sum_{i=1}^{n} \sum_{j \neq i} a_{i j}^{\prime} \cdot \Delta x_{i}^{\prime} \cdot \Delta x_{j}^{\prime} .
$$

After this calibration, the problem takes the following form: we know the intervals $\left[-\Delta_{i}^{-}, \Delta_{i}^{+}\right]$of possible values of $\Delta x_{i}^{\prime}$, we know that $E\left[\Delta x_{i}^{\prime}\right]=0$ for all $i$, we want to find the interval of possible values of $E[y]$.

What is currently known. The expression (1) (or (10)) is a linear combination of linear and quadratic terms $\Delta x_{i}$ and $\Delta x_{i} \cdot \Delta x_{j}$ (corr., $\Delta x_{i}^{\prime}$ and $\Delta x_{i}^{\prime} \cdot \Delta x_{j}^{\prime}$ ). The expected value of a linear combination is equal to the linear combination of the corresponding expected values. The expected values of $\Delta x_{i}$ and $\Delta x_{i}^{\prime}$ are known. So, to be able to estimate the expected value of $y$, it is sufficient to be able to estimate the expected value of a product $\Delta x_{i} \cdot \Delta x_{j}$.

It is known $[8,9]$ that if we have two random variables $v_{1}$ and $v_{2}$ with known ranges $\left[\underline{v}_{i}, \bar{v}_{i}\right]$ and known means $E_{i}$, then the interval $[\underline{E}, \bar{E}]$ of possible values of $E \stackrel{\text { def }}{=} E\left[v_{1} \cdot v_{2}\right]$ can be computed as follows. First, we compute the auxiliary values $p_{i} \stackrel{\text { def }}{=}\left(E_{i}-\underline{v}_{i}\right) /\left(\bar{v}_{i}-\underline{v}_{i}\right)$, and then compute

$$
\begin{gathered}
\underline{E}=\min \left(p_{1}+p_{2}-1,0\right) \cdot \bar{v}_{1} \cdot \bar{v}_{2}+\min \left(p_{1}, 1-p_{2}\right) \cdot \bar{v}_{1} \cdot \underline{v}_{2}+ \\
\min \left(1-p_{1}, p_{2}\right) \cdot \underline{v}_{1} \cdot \bar{v}_{2}+\max \left(1-p_{1}-p_{2}, 0\right) \cdot \underline{v}_{1} \cdot \underline{v}_{2} ; \\
\bar{E}=\min \left(1-p_{1}, 1-p_{2}\right) \cdot \underline{v}_{1} \cdot \underline{v}_{2}+\max \left(p_{1}-p_{2}, 0\right) \cdot \bar{v}_{1} \cdot \underline{v}_{2}+ \\
\max \left(p_{2}-p_{1}, 0\right) \cdot \underline{v}_{1} \cdot \bar{v}_{2}+\min \left(p_{1}, p_{2}\right) \cdot \bar{v}_{1} \cdot \bar{v}_{2} .
\end{gathered}
$$


In principle, we can use these formulas to estimate $E[y]$.

We can also apply a similar approach to the alternative quadratic formula (2). To use this approach, we need to know the means of the new variables $y_{i}$, but this is easy, since $y_{i}$ are linear combinations of $\Delta x_{i}$.

Remaining problem. In $[8,9]$, our objective was to come up with an expression for a single exact range of $E\left[v_{1} \cdot v_{2}\right]$ for two variables $v_{1}$ and $v_{2}$. The fact that we found an explicit analytical expression makes it easy to compute the range.

In our new problem, however, we need to estimate many $\left(\sim n^{2}\right)$ such ranges - because $n$ may be large. Each range computation requires two divisions (to compute $p_{1}$ and $p_{2}$ ) and several multiplications - and division is known to take longer to compute. Since we need to repeat these computations $\sim n^{2}$ times, it is desirable to look for simpler expressions for $\underline{E}$ and $\bar{E}$, expressions that would hopefully avoid division altogether and require fewer multiplications - and thus, will lead to faster computations. Such expressions are indeed possible:

Theorem 2 If we have two random variables $v_{1}$ and $v_{2}$ with known means $E_{i}$ and known ranges $\left[E_{i}-\Delta_{i}^{-}, E_{i}+\Delta_{i}^{+}\right]$, then the interval $[\underline{E}, \bar{E}]$ of possible values of $E=E\left[v_{1} \cdot v_{2}\right]$ is equal to

$$
\left[E_{1} \cdot E_{2}-\min \left(\Delta_{1}^{-} \cdot \Delta_{2}^{-}, \Delta_{1}^{+} \cdot \Delta_{2}^{+}\right), E_{1} \cdot E_{2}+\min \left(\Delta_{1}^{-} \cdot \Delta_{2}^{+}, \Delta_{1}^{+} \cdot \Delta_{2}^{-}\right)\right] .
$$

For the expression $v_{i}^{2}$, the range can be computed by using the general techniques from $[11,16]$. For readers' convenience, let us give an explicit derivation.

Theorem 3 If we have a random variable $v_{i}$ with a known mean $E_{i}$ and a known range $\left[E_{i}-\Delta_{i}^{-}, E_{i}+\Delta_{i}^{+}\right]$, then the interval $\left[\underline{M}_{i}, \bar{M}_{i}\right]$ of possible values of $M_{i}=E\left[v_{i}^{2}\right]$ is equal to $\left[E_{i}^{2}, E_{i}^{2}+\Delta_{i}^{-} \cdot \Delta_{i}^{+}\right]$.

We want to apply these theorems to the variables $v_{i}=\Delta x_{i}^{\prime}$ for which $E_{i}=$ $E\left[\Delta x_{i}^{\prime}\right]=0$; as a result, we get the following enclosure $\mathbf{E}_{\text {orig }}$ for the range $[\underline{E}, \bar{E}]$ of $E=E[y]$ takes the following form:

$$
\begin{gathered}
\mathbf{E}_{\text {orig }}=a_{0}^{\prime}-\sum_{i} a_{i i}^{\prime} \cdot\left[0, \Delta_{i}^{-} \cdot \Delta_{i}^{+}\right]+ \\
\sum_{i=1}^{n} \sum_{j \neq i} a_{i j}^{\prime} \cdot\left[-\min \left(\Delta_{i}^{-} \cdot \Delta_{j}^{+}, \Delta_{i}^{+} \cdot \Delta_{j}^{-}\right), \min \left(\Delta_{i}^{-} \cdot \Delta_{j}^{-}, \Delta_{i}^{+} \cdot \Delta_{j}^{+}\right)\right] .
\end{gathered}
$$

Alternatively, we can represent the matrix $a_{i j}^{\prime}$ in terms of its eigenvalues $\lambda_{k}^{\prime}$ and the corresponding unit eigenvectors $e_{k}^{\prime}=\left(e_{k 1}^{\prime}, \ldots, e_{k n}^{\prime}\right)$, then

$$
y=a_{0}^{\prime}+\sum_{i=1}^{n} a_{i}^{\prime} \cdot \Delta x_{i}^{\prime}+\sum_{k=1}^{n} \lambda_{k}^{\prime} \cdot\left(\sum_{i=1}^{n} e_{k i}^{\prime} \cdot \Delta x_{i}^{\prime}\right) .
$$


Each variable $\Delta x_{i}^{\prime}$ has 0 mean and range $\left[-\Delta_{i}^{-}, \Delta_{i}^{+}\right]$. Thus, for the linear combination $\sum_{i=1}^{n} e_{k i}^{\prime} \cdot \Delta x_{i}^{\prime}$, the mean is equal to 0 , and the range is

$$
\sum_{i=1}^{n} e_{k i}^{\prime} \cdot\left[-\Delta_{i}^{-}, \Delta_{i}^{+}\right]
$$

In other words, the range is equal to $\left[-\delta_{k}^{-}, \delta_{k}^{+}\right]$, where

$$
\begin{aligned}
& \delta_{k}^{-}=\sum_{i: e_{k i}^{\prime}<0}\left|e_{k i}^{\prime}\right| \cdot \Delta_{i}^{+}+\sum_{i: e_{k i}^{\prime}>0} e_{k i}^{\prime} \cdot \Delta_{i}^{-} \\
& \delta_{k}^{+}=\sum_{i: e_{k i}^{\prime}<0}\left|e_{k i}^{\prime}\right| \cdot \Delta_{i}^{-}+\sum_{i: e_{k i}^{\prime}>0} e_{k i}^{\prime} \cdot \Delta_{i}^{+} .
\end{aligned}
$$

As a result, we get a different enclosure $\mathbf{E}_{\text {new }}$ for the range $[\underline{E}, \bar{E}]$ of $E=E[f]$ :

$$
\mathbf{E}_{\text {new }}=a_{0}^{\prime}+\sum_{k=1}^{n} \lambda_{k} \cdot\left[0, \delta_{k}^{-} \cdot \delta_{k}^{+}\right] .
$$

It is worth mentioning that when $\Delta_{i}^{-}=\Delta_{i}^{+}$, both enclosures coincide with the enclosures corresponding to interval computations. Therefore, the results from the previous section not only describe which method is better for estimating the range of possible value of the desired quantity $y$, they also describe which method is better for estimating the mean of $y$.

Even faster computations are possible. According to Theorem 2, in general, to compute the range for $E$, it is sufficient to perform 5 multiplications: one to compute $E_{1} \cdot E_{2}$ and 4 to compute 4 products $\Delta_{1}^{ \pm} \cdot \Delta_{2}^{ \pm}$. In particular, for $v_{i}=\Delta x_{i}^{\prime}$, since we have $E_{i}=0$, we only need 4 multiplications.

It is possible to follow the ideas behind the fast algorithm for interval multiplication $[2,4]$ and reduce the number of multiplications by one.

For this, first, we compare $\Delta_{1}^{-}$with $\Delta_{1}^{+}$and $\Delta_{2}^{-}$with $\Delta_{2}^{+}$. As a result, we get $2 \times 2=4$ different comparison results. In all 4 cases, we can avoid at least one multiplication in the formula (13); indeed:

- if $\Delta_{1}^{-} \leq \Delta_{1}^{+}$and $\Delta_{2}^{-} \leq \Delta_{2}^{+}$, then $\min \left(\Delta_{1}^{-} \cdot \Delta_{2}^{-}, \Delta_{1}^{+} \cdot \Delta_{2}^{+}\right)=\Delta_{1}^{-} \cdot \Delta_{2}^{-}$;

- if $\Delta_{1}^{-} \leq \Delta_{1}^{+}$and $\Delta_{2}^{+} \leq \Delta_{2}^{-}$, then $\min \left(\Delta_{1}^{-} \cdot \Delta_{2}^{+}, \Delta_{1}^{+} \cdot \Delta_{2}^{-}\right)=\Delta_{1}^{-} \cdot \Delta_{2}^{+}$;

- if $\Delta_{1}^{+} \leq \Delta_{1}^{-}$and $\Delta_{2}^{-} \leq \Delta_{2}^{+}$, then $\min \left(\Delta_{1}^{-} \cdot \Delta_{2}^{+}, \Delta_{1}^{+} \cdot \Delta_{2}^{-}\right)=\Delta_{1}^{+} \cdot \Delta_{2}^{-}$;

- if $\Delta_{1}^{+} \leq \Delta_{1}^{-}$and $\Delta_{2}^{+} \leq \Delta_{2}^{-}$, then $\min \left(\Delta_{1}^{-} \cdot \Delta_{2}^{-}, \Delta_{1}^{+} \cdot \Delta_{2}^{+}\right)=\Delta_{1}^{+} \cdot \Delta_{2}^{+}$. 
Proof of Theorem 2. Let us start with the expression for $\bar{E}$. When $p_{1} \geq p_{2}$, the expression (12) takes the following simplified form:

$$
\bar{E}=p_{2} \cdot \bar{v}_{1} \cdot \bar{v}_{2}+\left(p_{1}-p_{2}\right) \cdot \bar{v}_{1} \cdot \underline{v}_{2}+\left(1-p_{1}\right) \cdot \underline{v}_{1} \cdot \underline{v}_{2} .
$$

Grouping together terms proportional to $p_{1}$ and $p_{2}$, we conclude that

$$
\bar{E}=p_{2} \cdot \bar{v}_{1} \cdot\left(\bar{v}_{2}-\underline{v}_{2}\right)+p_{1} \cdot\left(\bar{v}_{1}-\underline{v}_{1}\right) \cdot \underline{v}_{2}+\underline{v}_{1} \cdot \underline{v}_{2} .
$$

Substituting the expression defining $p_{i}$ into this formula, we conclude that

$$
\bar{E}=\bar{v}_{1} \cdot\left(E_{2}-\underline{v}_{2}\right)+\left(E_{1}-\underline{v}_{1}\right) \cdot \underline{v}_{2}+\underline{v}_{1} \cdot \underline{v}_{2} .
$$

Grouping the last two terms, we get

$$
\bar{E}=\bar{v}_{1} \cdot\left(E_{2}-\underline{v}_{2}\right)+E_{1} \cdot \underline{v}_{2} .
$$

Finally, substituting $\underline{v}_{i}=E_{i}-\Delta_{i}^{-}$and $\bar{v}_{i}=E_{i}+\Delta_{i}^{+}$, we conclude that

$\bar{E}=\left(E_{1}+\Delta_{1}^{+}\right) \cdot \Delta_{2}^{-}+E_{1} \cdot\left(E_{2}-\Delta_{2}^{-}\right)=E_{1} \cdot \Delta_{2}^{-}+\Delta_{1}^{+} \cdot \Delta_{2}^{-}+E_{1} \cdot E_{2}-E_{1} \cdot \Delta_{2}^{-}$, i.e.,

$$
\bar{E}=E_{1} \cdot E_{2}+\Delta_{1}^{+} \cdot \Delta_{2}^{-} .
$$

Similarly, if $p_{1} \leq p_{2}$, we get

$$
\bar{E}=E_{1} \cdot E_{2}+\Delta_{1}^{-} \cdot \Delta_{2}^{+} .
$$

The condition $p_{1} \geq p_{2}$, i.e., $\left(E_{1}-\underline{v}_{1}\right) /\left(\bar{v}_{1}-\underline{v}_{1}\right) \geq\left(E_{2}-\underline{v}_{2}\right) /\left(\bar{v}_{2}-\underline{v}_{2}\right)$, can be equivalently described as

$$
\left(E_{1}-\underline{v}_{1}\right) \cdot\left(\bar{v}_{2}-\underline{v}_{2}\right) \geq\left(E_{2}-\underline{v}_{2}\right) \cdot\left(\bar{v}_{1}-\underline{v}_{1}\right),
$$

i.e., in terms of $E_{i}$ and $\Delta_{i}^{ \pm}$, as

$$
\Delta_{1}^{-} \cdot\left(\Delta_{2}^{-}+\Delta_{2}^{+}\right) \geq \Delta_{2}^{-} \cdot\left(\Delta_{1}^{-}+\Delta_{1}^{+}\right),
$$

or, equivalently, as

$$
\Delta_{1}^{-} \cdot \Delta_{2}^{+} \geq \Delta_{1}^{+} \cdot \Delta_{2}^{-} .
$$

Since this condition determines whether we have an expression (23) or (24), we thus get the desired formula for $\bar{E}$.

For $\underline{E}$, we similarly consider two cases: $p_{1}+p_{2} \geq 1$ and $p_{1}+p_{2}<1$. In the first case, we have

$$
\underline{E}=\left(p_{1}+p_{2}-1\right) \cdot \bar{v}_{1} \cdot \bar{v}_{2}+\left(1-p_{2}\right) \cdot \bar{v}_{1} \cdot \underline{v}_{2}+\left(1-p_{1}\right) \cdot \underline{v}_{1} \cdot \bar{v}_{2},
$$

i.e.,

$$
\underline{E}=p_{1} \cdot\left(\bar{v}_{1}-\underline{v}_{1}\right) \cdot \bar{v}_{2}+p_{2} \cdot \bar{v}_{1} \cdot\left(\bar{v}_{2}-\underline{v}_{2}\right)+
$$




$$
\underline{v}_{1} \cdot \bar{v}_{2}+\bar{v}_{1} \cdot \underline{v}_{2}-\bar{v}_{1} \cdot \bar{v}_{2} .
$$

Substituting the expressions for $p_{i}$, we conclude that

$$
\underline{E}=\left(E_{1}-\underline{v}_{1}\right) \cdot \bar{v}_{2}+\bar{v}_{1} \cdot\left(E_{2}-\underline{v}_{2}\right)+\underline{v}_{1} \cdot \bar{v}_{2}+\bar{v}_{1} \cdot \underline{v}_{2}-\bar{v}_{1} \cdot \bar{v}_{2},
$$

i.e.,

$$
\underline{E}=E_{1} \cdot \bar{v}_{2}+\bar{v}_{1} \cdot E_{2}-\bar{v}_{1} \cdot \bar{v}_{2} .
$$

Finally, substituting the expressions $v_{i}=E_{i}-\Delta_{i}^{-}$and $\bar{v}_{i}=E_{i}+\Delta_{i}^{+}$, we conclude that

$$
\underline{E}=E_{1} \cdot E_{2}-\Delta_{1}^{+} \cdot \Delta_{2}^{+} .
$$

Similarly, if $p_{1}+p_{2}<1$, then

$$
\underline{E}=p_{1} \cdot \bar{v}_{1} \cdot \underline{v}_{2}+p_{2} \cdot \underline{v}_{1} \cdot \bar{v}_{2}+\left(1-p_{1}-p_{2}\right) \cdot \underline{v}_{1} \cdot \underline{v}_{2},
$$

i.e.,

$$
\underline{E}=p_{1} \cdot\left(\bar{v}_{1}-\underline{v}_{1}\right) \cdot \underline{v}_{2}+p_{2} \cdot \underline{v}_{1} \cdot\left(\bar{v}_{2}-\underline{c}_{2}\right)+\underline{v}_{1} \cdot \underline{v}_{2} .
$$

Substituting the expressions for $p_{i}$, we conclude that

$$
\underline{E}=\left(E_{1}-\underline{v}_{1}\right) \cdot \underline{v}_{2}+\underline{v}_{1} \cdot\left(E_{2}-\underline{v}_{2}\right)+\underline{v}_{1} \cdot \underline{v}_{2},
$$

i.e., that

$$
\underline{E}=E_{1} \cdot \underline{v}_{2}+\underline{v}_{1} \cdot\left(E_{2}-\underline{v}_{2}\right) .
$$

Finally, substituting the expressions $v_{i}=E_{i}-\Delta_{i}^{-}$and $\bar{v}_{i}=E_{i}+\Delta_{i}^{+}$, we conclude that

$$
\underline{E}=E_{1} \cdot E_{2}-\Delta_{1}^{-} \cdot \Delta_{2}^{-} .
$$

The inequality $p_{1}+p_{2} \geq 1$ can be reformulated as

$$
\left(E_{1}-\underline{v}_{1}\right) \cdot\left(\bar{v}_{2}-\underline{v}_{2}\right)+\left(E_{2}-\underline{v}_{2}\right) \cdot\left(\bar{v}_{1}-\underline{v}_{1}\right) \geq\left(\bar{v}_{1}-\underline{v}_{1}\right) \cdot\left(\bar{v}_{2}-\underline{v}_{2}\right),
$$

i.e., subtracting the first product from both sides, as

$$
\left(E_{2}-\underline{v}_{2}\right) \cdot\left(\bar{v}_{1}-\underline{v}_{1}\right) \geq\left(\bar{v}_{1}-E_{1}\right) \cdot\left(\bar{v}_{2}-\underline{v}_{2}\right),
$$

or, in terms of $\Delta_{i}^{ \pm}$, as

$$
\Delta_{2}^{-} \cdot\left(\Delta_{1}^{-}+\Delta_{1}^{+}\right) \geq \Delta_{1}^{+} \cdot\left(\Delta_{2}^{-}+\Delta_{2}^{+}\right)
$$

i.e., equivalently,

$$
\Delta_{1}^{-} \cdot \Delta_{2}^{-} \geq \Delta_{1}^{+} \cdot \Delta_{2}^{+}
$$

Since this condition determines whether we have an expression (31) or (36), we thus get the desired formula for $\bar{E}$.

The theorem is proven. 
Proof of Theorem 3. It is known that the second moment $M_{i}$ is equal to $V_{i}+E_{i}^{2}$, where $V_{i}$ is the variance, i.e., the second moment of the auxiliary variable $v \stackrel{\text { def }}{=} v_{i}-E_{i}$ for which $E[v]=0$ and for which the range of possible values is equal to $\left[-\Delta_{i}^{-}, \Delta_{i}^{+}\right]$. Thus, to prove the theorem, it is sufficient to prove that if we have a random variable $v$ with a known mean $E[v]=0$ and a known range $\left[-\Delta^{-}, \Delta^{+}\right]$, then the interval $[\underline{M}, \bar{M}]$ of possible values of $M=E\left[v^{2}\right]$ is equal to $\left[0, \Delta^{-} \cdot \Delta^{+}\right]$.

The second moment is always non-negative, so $\underline{M} \geq 0$. It is possible that $v$ is identically 0 , in which case $M=0$; thus, $\underline{M}=0$.

Among all distributions with 0 mean located on the interval $\left[-\Delta^{-}, \Delta^{+}\right]$, we want to find a distribution for which the second moment is the largest. For discrete distributions, that attains values $x_{1}, \ldots, x_{n}$ with probabilities $p_{1}, \ldots, p_{n}$, this means that we must maximize the expression $\sum p_{i} \cdot x_{i}^{2}$ under the constraints $p_{i} \geq 0, \sum p_{i}=1$, and $\sum p_{i} \cdot x_{i}=1$.

Once the values $x_{i}$ are fixed, this constraint optimization problem becomes a linear programming problem with 2 equality constraints; according to the general properties of linear programming problems problems, the maximum is attained when at most 2 of the variables $p_{i}$ are non-zero (see [8] for detailed description). Thus, to find the value $\bar{M}$, it is sufficient to consider distributions located at only two points $x_{1}$ and $x_{2}$. Since the average is 0 , one of these values should be negative, and another positive. Let us denote the negative value by $-x^{-}$and the positive one by $x^{+}$, and the corresponding probabilities by $p^{-}$and $p^{+}$.

Since $p^{-}+p^{+}=1$, we get $p^{+}=1-p^{-}$. Thus, from the condition

$$
p^{-} \cdot\left(-x^{-}\right)+\left(1-p^{-}\right) \cdot x^{+}=0,
$$

i.e., equivalently, $p^{-}\left(x^{-}+x^{+}\right)=x^{+}$, we conclude that $p^{-}=x^{+} /\left(x^{-}+x^{+}\right)-$ and hence, that $p^{+}=1-p^{-}=x^{-} /\left(x^{-}+x^{+}\right)$. Therefore,

$$
\begin{gathered}
M=p^{-} \cdot\left(x^{-}\right)^{2}+p^{+} \cdot\left(x^{+}\right)^{2}= \\
\frac{x^{+}}{x^{-}+x^{+}} \cdot\left(x^{-}\right)^{2}+\frac{x^{-}}{x^{-}+x^{+}} \cdot\left(x^{+}\right)^{2}=\frac{x^{-} \cdot x^{+} \cdot\left(x^{-}+x^{+}\right)}{x^{-}+x^{+}}=x^{-} \cdot x^{+} .
\end{gathered}
$$

This expression is a strictly increasing function of both its variables $x^{-}, x^{+} \geq 0$. Thus, its maximum under the constraints $x^{-} \leq \Delta^{-}$and $x^{+} \leq \Delta^{+}$is attained when $x^{-}=\Delta^{-}$and $x^{+}=\Delta^{+}$, and the corresponding value is exactly the one we described. The theorem is proven.

What if measurements are actually independent? In the above text, we assumed that the measurement errors $\Delta x_{i}$ can be correlated, and we have no information about possible correlations. In some cases, however, we do know that the variables $\Delta x_{i}$ are independent. Under this additional assumption of independence, what is the possible range of $E[y]$ ? 
This question is the easiest to answer under the formula (10). Indeed, in this case, $E\left[\Delta x_{i}^{\prime}\right]=0$, and for $i \neq j$, we have $E\left[\Delta x_{i}^{\prime} \cdot \Delta x_{j}^{\prime}\right]=E\left[\Delta x_{i}^{\prime}\right] \cdot E\left[\Delta x_{j}^{\prime}\right]=$ $0 \cdot 0=0 ;$ so, we conclude that

$$
E=E[y]=a_{0}^{\prime}+\sum_{i=1}^{n} a_{i i}^{\prime} \cdot E\left[\left(\Delta x_{i}^{\prime}\right)^{2}\right] .
$$

From Theorem 3, we already know how to compute the range of possible values of $E\left[\left(\Delta x_{i}^{\prime}\right)^{2}\right]$ for $i=1, \ldots, n$. So, in the case of independence, the interval of possible values of $E$ is equal to

$$
[\underline{E}, \bar{E}]=a_{0}^{\prime}+\sum_{i=1}^{n} a_{i i}^{\prime} \cdot\left[0, \Delta_{i}^{-} \cdot \Delta_{i}^{+}\right] .
$$

This is the exact range, so there is no need for using eigenvectors and eigenvalues.

Remaining open problem. In practice, in addition to the first moments $E\left[\Delta x_{i}\right]$, we often know the second moments $E\left[\Delta x_{i} \cdot \Delta x_{j}\right]$ of the corresponding distributions. If we know the second moments, then, of course, computing the first moment of the quadratic expression $y$ is easy, since $y$ is a linear combination of terms $\Delta x_{i}$ and $\Delta x_{i} \cdot \Delta x_{j}$. However, in this case, a natural next question remains open: what can we say about the second moment of $y$ ?

\section{Acknowledgments.}

This work was supported in part by NASA grant NCC5-209, by the Air Force Office of Scientific Research grant F49620-00-1-0365, by NSF grants EAR-0112968, EAR-0225670, and EIA-0321328, and by the Army Research Laboratories grant DATM-05-02-C-0046. This work was partly performed during V. Kreinovich's visit to Brazil. This visit was sponsored by the Brazilian funding agency CTINFO/CNPq.

The authors are thankful to all the participants of the Workshop on State-ofthe-Art in Scientific Computing PARA'04, Lyngby, Denmark (June 20-23, 2004) for fruitful discussion, and to the anonymous referees for valuable suggestions.

\section{References}

[1] Ferson, S.: RAMAS Risk Calc 4.0, CRC Press, Boca Raton, Florida, 2002.

[2] Hamzo, C., Kreinovich, V.: On Average Bit Complexity of Interval Arithmetic, Bulletin of the European Association for Theoretical Computer Science (EATCS), 1999, Vol. 68, pp. 153-156.

[3] Hansen, E.: Sharpness in interval computations, Reliable Computing, 1997, Vol. 3, pp. 7-29. 
[4] Heindl, G.: An improved algorithm for computing the product of two machine intervals, Interner Bericht IAGMPI- 9304, Fachbereich Mathematik, Gesamthochschule Wuppertal, 1993.

[5] Jaulin, L., Kieffer, M., Didrit, O., Walter, E.: Applied Interval Analysis: With Examples in Parameter and State Estimation, Robust Control and Robotics, Springer, London, 2001.

[6] Kearfott, R.B.: Rigorous Global Search: Continuous Problems. Kluwer, Dordrecht, 1996.

[7] Koltik, E., Dmitriev, V.G., Zheludeva, N.A., Kreinovich, V.: An optimal method for estimating a random error component, Investigations in Error Estimation, Proceedings of the Mendeleev Metrological Institute, Leningrad, 1986, 36-41 (in Russian).

[8] Kreinovich, V.: Probabilities, Intervals, What Next? Optimization Problems Related to Extension of Interval Computations to Situations with Partial Information about Probabilities, Journal of Global Optimization, 2004, Vol. 29, No. 3, pp. 265-280.

[9] Kreinovich, V., Ferson, S., and Ginzburg, L.: Exact Upper Bound on the Mean of the Product of Many Random Variables With Known Expectations, Reliable Computing, 2003, Vol. 9, No. 6, pp. 441-463.

[10] Kreinovich, V., Lakeyev, A., Rohn, J., Kahl, P.: Computational Complexity and Feasibility of Data Processing and Interval Computations, Kluwer, Dordrecht, 1997.

[11] Kuznetsov, V. P.: Interval Statistical Models, Radio i Svyaz Publ., Moscow, 1991 (in Russian).

[12] Moore, R.E.: Methods and Applications of Interval Analysis. SIAM, Philadelphia, 1979.

[13] Rabinovich, S.: Measurement Errors: Theory and Practice, American Institute of Physics, New York, 1993.

[14] Trejo, R., Kreinovich, V.: Error Estimations for Indirect Measurements: Randomized vs. Deterministic Algorithms For "Black-Box" Programs, In: Rajasekaran, S., Pardalos, P., Reif, J., Rolim, J., eds., Handbook on Randomized Computing, Kluwer, 2001, 673-729.

[15] Vavasis, S.A.: Nonlinear Optimization: Complexity Issues, Oxford University Press, New York, 1991.

[16] Walley, P.: Statistical Reasoning with Imprecise Probabilities, Chapman \& Hall, N.Y., 1991. 